Discrete Comput Geom 26:283-287 (2001)

DOI: $10.1007 / \mathrm{s} 00454-001-0037-8$

\title{
Lower Bounds on the Transversal Numbers of $d$-Intervals*
}

\author{
J. Matoušek \\ Department of Applied Mathematics, Charles University, \\ Malostranské nám. 25, 11800 Praha 1, Czech Republic \\ matousek@kam.mff.cuni.cz
}

\begin{abstract}
Let $\ell_{1}, \ell_{2}, \ldots, \ell_{d}$ be disjoint parallel lines in the plane. A $d$-interval is a subset of their union that intersects each $\ell_{i}$ in a closed interval. Kaiser [4] showed that any system of $d$-intervals containing no subsystem of $k+1$ pairwise disjoint $d$-intervals can be pierced by at most $\left(d^{2}-d\right) k$ points. We show that this bound is close to being optimal, by proving a lower bound of const $\left(d^{2} / \log ^{2} d\right) k$. The construction involves an extension of a construction due to Sgall [8] of certain systems of set pairs.
\end{abstract}

\section{Introduction}

Let $\ell_{1}, \ell_{2}, \ldots, \ell_{d}$ be disjoint parallel lines in the plane. A set $J \subset \bigcup_{j=1}^{d} \ell_{j}$ is a $d$-interval if it intersects each $\ell_{i}$ in a closed interval. A homogeneous $d$-interval is the union of at most $d$ closed intervals on the real line.

Let $\mathcal{S}$ be a system of subsets of a ground set $X$. The matching number $v(\mathcal{S})$ is the maximum cardinality of a subsystem $\mathcal{M} \subseteq \mathcal{S}$ consisting of pairwise disjoint sets. The transversal number $\tau(\mathcal{S})$ is the smallest possible cardinality of a transversal of $\mathcal{S}$, i.e., of a subset $T \subseteq X$ with $S \cap T \neq \emptyset$ for all $S \in \mathcal{S}$. For $d$-intervals, the matching number and transversal number are taken in this set-theoretical sense.

Gyárfás and Lehel [2] (also see [3]) initiated the investigation of the following question. Let $\mathcal{F}$ be a family of $d$-intervals with $v(\mathcal{F}) \leq k$. What is the maximum possible value of $\tau(\mathcal{F})$, in terms of $k$ and $d$ ? (Note that for $d=1$, we have $\nu(\mathcal{F})=\tau(\mathcal{F})$ for any family of 1-intervals, by a well-known property of systems of intervals.) They proved that there is a finite bound for all $k$ and $d$; their estimate was rather large, about $k^{d !}$ for a fixed $d$. Károlyi and Tardos [6] obtained a bound of $O\left(k^{d}\right)$ for every fixed $d$. A major breakthrough was achieved by Tardos [9], who proved $\tau(\mathcal{F}) \leq 2 v(\mathcal{F})$ for any family

\footnotetext{
* This research was supported by Charles University Grants Nos. 158/99 and 159/99 and by the Grant
} KONTAKT 338/1999. 
of 2-intervals (which is tight in the worst case) by an ingenious topological method. By a somewhat different topological approach, Kaiser [4] proved that for any system of $d$ intervals with $v(\mathcal{F}) \leq k$, we have $\tau(\mathcal{F}) \leq\left(d^{2}-d\right) k$. Later Alon [1] found a short nontopological proof of the slightly weaker bound $\tau(\mathcal{F}) \leq 2 d^{2} k$.

Homogeneous $d$-intervals are strictly more general than $d$-intervals, and the upper bounds obtained for them are slightly worse: Kaiser [4] showed $\tau(\mathcal{F}) \leq 3 k$ for any family of homogeneous 2-intervals with $v(\mathcal{F}) \leq k$, which is the best possible, and $\tau(\mathcal{F}) \leq\left(d^{2}-d+1\right) k$ for an arbitrary family of homogeneous $d$-intervals with $v(\mathcal{F}) \leq k$, for any $d \geq 3$. Alon's upper bound applies for homogeneous $d$-intervals as well.

The best lower bound up until now was given by Kaiser [5], who constructed an example with $v=k$ and $\tau \geq 2(d-1) k$, for all $d$ and $k$, improving a bound of $d k$ of Károlyi and Tardos [6]. Here we give lower bounds which are near-quadratic in $d$ and almost match Kaiser's upper bounds.

Theorem 1.1. There is a positive constant $c>0$ such that:

(i) For any $d \geq 1$ and $k \geq 1$, there is a family $\mathcal{F}$ of homogeneous $d$-intervals with $v(\mathcal{F})=k$ and

$$
\tau(\mathcal{F}) \geq c \frac{d^{2}}{\log d} k .
$$

(ii) For any $d \geq 1$ and $k \geq 1$, there is a family $\mathcal{F}$ of $d$-intervals with $v(\mathcal{F})=k$ and

$$
\tau(\mathcal{F}) \geq c \frac{d^{2}}{(\log d)^{2}} k
$$

Part (i) follows in a simple way from an ingenious construction due to Sgall [8] of certain systems of set pairs. For part (ii), we need to modify Sgall's construction to yield $d$-partite set systems. The families of (homogeneous) $d$-intervals constructed in the proof of Theorem 1.1 are of a fairly restricted type: each $d$-interval consists of points and of unit-length intervals, and any two unit-length intervals appearing as a component of a $d$-interval in the construction are either disjoint or identical.

The most interesting related open problem, in our opinion, is to estimate the maximum possible transversal number of a system of (homogeneous) $d$-intervals in which every three $d$-intervals have a common point, as a function of $d$. Is it (almost) linear, nearquadratic, or somewhere in between (perhaps like $d^{3 / 2}$ )?

\section{Sgall's Construction and Homogeneous $d$-Intervals}

Wigderson raised a problem in 1985 which can be reformulated as follows. Suppose that $A_{1}, A_{2}, \ldots, A_{N}$ and $B_{1}, B_{2}, \ldots, B_{N}$ are sets with the following properties:

(C1) for all $i=1,2, \ldots, N, \emptyset \neq B_{i} \subseteq A_{i}$ and $\left|A_{i}\right| \leq a$, and

(C2) for all $i_{1}, i_{2}, 1 \leq i_{1}<i_{2} \leq N$, we have $A_{i_{1}} \cap B_{i_{2}} \neq \emptyset$ or $A_{i_{2}} \cap B_{i_{1}} \neq \emptyset$.

Let $\mathcal{B}=\left\{B_{i}: i=1,2, \ldots, N\right\}$. What is the maximum possible value of $\tau(\mathcal{B})$, in terms of $a$ ? 
It is not difficult to see that $\tau(\mathcal{B}) \leq a(a+1) / 2$ : Put all points of $A_{1}$ into the transversal, remove them from all the $A_{i}$ and $B_{i}$, and discard all $i$ with $B_{i} \cap A_{1} \neq \emptyset$; for the remaining $i$, we now have $\left|A_{i}\right| \leq a-1$ and we can use induction on $a$. A clever construction by Sgall [8] shows that this simple bound cannot be improved much and that $\tau(\mathcal{B})$ can be as large as const $\cdot a^{2} / \log a$. (The title of Sgall's paper refers to a different, but essentially equivalent, formulation of the problem dealing with labeled tournaments.) For completeness and for the reader's delight, we briefly recall the construction, although we do not need it.

Set $b=\lfloor a / 3\rfloor$ and $n=c b^{2} / \log b$ for a sufficiently small constant $c>0$. Let $V$ be an $n$-element ground set. Let $G=(V, E)$ be a graph of maximum degree $b$ with the following expander-type property: for any two disjoint $b$-element subsets $A, B \subseteq V$, there is at least one edge $e \in E$ connecting a vertex of $A$ to a vertex of $B$ (the existence of such a graph can be easily shown by the probabilistic method; see [8] for references). Let $\mathcal{B}=\left\{B_{1}, B_{2}, \ldots, B_{N}\right\}$ be a system of $b$-element subsets of $V$ with $\tau(\mathcal{B})=t$ (taking all $b$-tuples for $\mathcal{B}$, we have $t=n-b+1)$. For each $i$, let $v_{i}$ be an (arbitrary) element of $B_{i}$, and let $A_{i}=B_{i} \cup N\left(v_{i}\right) \cup\left(V \backslash \bigcup_{u \in B_{i}} N(u)\right)$, where $N(v)$ denotes the set of neighbors in $G$ of a vertex $v \in V$. It is easy to check that $\left|A_{i}\right| \leq 3 b$, and some thought reveals that condition $(\mathrm{C} 2)$ is satisfied.

Proof of part (i) of Theorem 1.1. Given $d$ and $k$, we want to construct a system $\mathcal{F}$ of homogeneous $d$-intervals. Clearly, it suffices to consider the case $k=1$, since for larger $k$, we can take $k$ disjoint copies of the $\mathcal{F}$ constructed for $k=1$. Thus, we want an $\mathcal{F}$ in which every two $d$-intervals intersect and with $\tau(\mathcal{F})$ large.

Let $A_{1}, A_{2}, \ldots, A_{N}$ and $B_{1}, B_{2}, \ldots, B_{N}$ be sets satisfying (C1) with $a=d$ and (C2) and such that $\tau(\mathcal{B}) \geq t=c d^{2} / \log d$ (where, as before, $\mathcal{B}=\left\{B_{1}, \ldots, B_{N}\right\}$ ). We note that if we remove any at most $t / 2$ sets of $\mathcal{B}$, the resulting set system still has a transversal number at least $t / 2$.

Let $V=\bigcup_{i=1}^{N} A_{i}$ be the ground set. For each $v \in V$, choose a unit interval $I_{v} \subset \mathbf{R}$, in such a way that $I_{v} \cap I_{v^{\prime}}=\emptyset$ for $v \neq v^{\prime}$. For each $v \in V$ and $i=1,2, \ldots, N$, choose a point $x_{v, i} \in I_{v}$, taking distinct points for distinct $i$. Define a system $\mathcal{J}$ of $N$ homogeneous $d$-intervals $J_{1}, J_{2}, \ldots, J_{N}$ by setting

$$
J_{i}=\left(\bigcup_{v \in B_{i}} I_{v}\right) \cup\left\{x_{u, i}: u \in A_{i} \backslash B_{i}\right\} .
$$

Condition (C2) implies that $\mathcal{J}$ is intersecting: if, for example, $u \in A_{i_{1}} \cap B_{i_{2}}$, then $x_{u, i_{1}} \in J_{i_{1}} \cap J_{i_{2}}$.

Suppose that $T$ is a transversal of $\mathcal{J}$. At most $|T|$ of the $J_{i}$ are intersected by $T$ in their point components, and so the set $\left\{v \in V: T \cap I_{v} \neq \emptyset\right\}$ is a transversal for some system of all but at most $|T|$ sets among the $B_{i}$. It follows that $2|T| \geq t=c d^{2} / \log d$.

Remark. It would be interesting to narrow the gap of $\log d$ between the lower and upper bounds, at least in Wigderson's problem about set pairs. The number of vertices $n=$ const $\cdot b^{2} / \log b$ for the expander graph in Sgall's construction is asymptotically the largest possible, as was communicated to me by Noga Alon. This follows by the Kôvári-Sós-Turán bound [7] for the maximum number of edges of a $K_{b, b}$-free graph on $n$ vertices applied to the complement of $G$. 


\section{Making the Construction $d$-Partite}

Call a set system $\mathcal{S}$ on a ground set $V d$-partite if $V$ can be partitioned into disjoint subsets $V_{1}, V_{2}, \ldots, V_{d}$ such that $\left|V_{j} \cap S\right| \leq 1$ for all $S \in \mathcal{S}$ and all $j$. Part (ii) of Theorem 1.1 is proved by constructing set systems $\overline{\mathcal{A}}=\left\{\bar{A}_{1}, \bar{A}_{2}, \ldots, \bar{A}_{N}\right\}$ and $\overline{\mathcal{B}}=\left\{\bar{B}_{1}, \bar{B}_{2}, \ldots, \bar{B}_{N}\right\}$ with properties as in Sgall's construction but also $d$-partite, for a suitable $d$ not much larger than $a$. We transform the sets from Sgall's construction using the following lemma.

Lemma 3.1 (On $m$-Partite Representation). Let $V$ be an $n$-element set, and let $a \leq n$ be a parameter. Then there are integers $m=O(a \log n)$ and $r=O(\log n)$ such that the following exist:

- a set $W$ partitioned into disjoint subsets $W_{1}, W_{2}, \ldots, W_{m}$,

- for each $v \in V$, an at most $(2 r-1)$-element set $U_{v} \subseteq W$ such that $U_{v} \cap U_{v^{\prime}}=\emptyset$ for $v \neq v^{\prime}$,

- for each $A \subseteq V$ with $|A| \leq a$, a set $f(A) \subseteq \bigcup_{v \in A} U_{v}$, such that $\left|f(A) \cap W_{j}\right| \leq 1$ for all $j$ and such that, for any $v \in A,\left|f(A) \cap U_{v}\right| \geq r$.

Proof of part (ii) of Theorem 1.1 (assuming Lemma 3.1). As in part (i), it suffices to consider the case $k=1$. Set $a=\left\lfloor c_{1} d / \log d\right\rfloor$ for a sufficiently small constant $c_{1}>0$, let $b=\lfloor a / 3\rfloor$, and let $n=c b^{2} / \log b$. Let $B_{1}, B_{2}, \ldots, B_{N}$ be all the $b$-tuples on an $n$-element ground set $V$, and let $A_{1}, A_{2}, \ldots, A_{N}$ be the at most $a$-element sets as in Sgall's construction.

We consider $W=W_{1} \dot{\cup} W_{2} \dot{\cup} \ldots \dot{U} W_{m}$, the $U_{v}$, and the $f(A)$ as in Lemma 3.1; we may assume $m=d$. Let $\bar{A}_{i}=f\left(A_{i}\right)$ and $\bar{B}_{i}=\bar{A}_{i} \cap\left(\bigcup_{v \in B_{i}} U_{v}\right) \subseteq \bar{A}_{i}$. The system $\overline{\mathcal{A}}=\left\{\bar{A}_{i}: i=1,2, \ldots, N\right\}$ is $d$-partite. If $v \in A_{i_{1}} \cap B_{i_{2}}$, then $\left|\bar{A}_{i_{1}} \cap U_{v}\right| \geq r$ and $\left|\bar{B}_{i_{2}} \cap U_{v}\right| \geq r$, and since $\left|U_{v}\right| \leq 2 r-1$, we have $\bar{A}_{i_{2}} \cap \bar{B}_{i_{2}} \neq \emptyset$. So (C2) holds for the $\bar{A}_{i}$ and $\bar{B}_{i}$. Moreover, the transversal number for any subsystem of $\overline{\mathcal{B}}$ is no smaller than the transversal number for the corresponding subsystem of $\mathcal{B}$.

It remains to construct the system $\mathcal{J}=\left\{J_{1}, J_{2}, \ldots, J_{N}\right\}$ of $d$-intervals. This is the same as in the proof of part (i), only this time we choose disjoint unit intervals $I_{w} \subset$ $\ell_{j}$ for $w \in W_{j}, j=1,2, \ldots, d$. The resulting $\tau(\mathcal{J})$ is at least const $\cdot b^{2} / \log b=$ const $\cdot d^{2} / \log ^{2} d$. Part (ii) of Theorem 1.1 is proved.

Proof of Lemma 3.1. Choose $m$ and $r$ so that there exists a bipartite graph $H$ (expander) with color classes $V$ and $[m]=\{1,2, \ldots, m\}$ with the following properties:

(a) Each vertex $v \in V$ has at most $2 r-1$ neighbors in $[m]$.

(b) For any subset $A \subseteq V$ with $|A| \leq a$, the number of (distinct) neighbors of $A$ in $[m]$ is at least $r|A|$.

The existence of such an expander with $r=O(\log n)$ and $m=O(a r)$ follows by a standard probabilistic argument, and it might very well be mentioned in the literature. Since we have no explicit reference, the calculation is sketched here.

For each $v \in V$, pick its neighbors in $[m]$ by $(2 r-1)$ independent random draws (with repetitions allowed, so we have at most $(2 r-1)$-neighbors but possibly fewer). 
For a fixed $s$-element $A \subseteq V$ and an (sr)-element $T \subseteq[m]$, the probability that all neighbors of $A$ live in $T$ is $(s r / m)^{(2 r-1) s}$. Thus, the probability that a random $H$ violates (b) is at most

$$
\begin{aligned}
\sum_{s=1}^{a}\left(\begin{array}{l}
n \\
s
\end{array}\right)\left(\begin{array}{l}
m \\
s r
\end{array}\right)\left(\frac{s r}{m}\right)^{(2 r-1) s} & \leq \sum_{s=1}^{a} n^{s}\left(\frac{e m}{s r}\right)^{s r}\left(\frac{s r}{m}\right)^{(2 r-1) s} \\
& =\sum_{s=1}^{a}\left[e n\left(\frac{e s r}{m}\right)^{r-1}\right]^{s}
\end{aligned}
$$

With $m=\lceil 2 e a r\rceil$ and $r=4+\left\lceil\log _{2} n\right\rceil$, say, the expression in square brackets is below $\frac{1}{2}$ and so random $H$ works with a positive probability.

Given a graph $H$ satisfying (a) and (b), we let $W$ be the set of edges of $H$. For $j \in[m]$, $W_{j}$ are the edges incident to $j$, and for $v \in V, U_{v}$ are the at most $2 r-1$ edges incident to $v$.

Consider an $A \subseteq V$ of size at most $a$. By condition (b), any $s$ vertices in $A$ have together at least $r s$ neighbors in $[m]$. By an $r$-fold application of Hall's marriage theorem, we can select an $r$-fold system of distinct representatives for the $a$ neighborhoods of the vertices $v \in A$. That is, for each $v \in A$, we choose a subset $U_{v, A} \subseteq U_{v}$ of $r$ edges incident to $v$, in such a way that no two edges in $\bigcup_{v \in A} U_{v, A}$ share an endpoint in $[m]$. We set $f(A)=\bigcup_{v \in A} U_{v, A}$. The conditions of Lemma 3.1 are satisfied.

\section{Acknowledgments}

I would like to thank Noga Alon and Nati Linial for pointing out Sgall's paper to me, in response to my question about set pairs discussed above. I also thank the referees for many helpful comments.

\section{References}

1. N. Alon. Piercing $d$-intervals. Discrete Comput. Geom., 19:333-334, 1998

2. A. Gyárfás and J. Lehel. A Helly-type problem in trees. In: Combinatorial Theory and its Applications (P. Erdôs et al. eds.), Colloquia Math. Soc. Janos Bolyai 4, North-Holland, Amsterdam, 1970, pages 571584.

3. A. Gyárfás and J. Lehel. Covering and coloring problems for relatives of intervals. Discrete Math., 55:167180,1985

4. T. Kaiser. Transversals of $d$-intervals. Discrete Comput. Geom., 18:195-203, 1997.

5. T. Kaiser. Piercing problems and topological methods. Doctoral dissertation, Department of Applied Mathematics, Charles University, Prague, 1998.

6. Gy. Károlyi and G. Tardos. On point covers of multiple intervals and axis-parallel rectangles. Combinatorica, 16:213-222, 1996.

7. T. Kővári, V. T. Sós, and P. Turán. On a problem of K. Zarankiewicz. Colloq. Math., 3:50-57, 1954.

8. J. Sgall. Solution of a covering problem related to labelled tournaments. J. Graph Theory, 23:111-118, 1996.

9. G. Tardos. Transversals of 2-intervals, a topological approach. Combinatorica, 15:123-134, 1995.

Received April 4, 2000, and in revised form January 4, 2001. Online publication August 28, 2001. 